\title{
Existence and uniqueness of positive solutions to boundary value problem with increasing homeomorphism and positive homomorphism operator
}

Fenghua Miao ${ }^{1}$, Chenxing Zhou ${ }^{1}$ and Yueqiang Song ${ }^{2^{*}}$

*Correspondence:

songyueqiang@sohu.com

${ }^{2}$ Scientific Research Department,

Changchun Normal University,

Changchun, Jilin 130032, PR China

Full list of author information is

available at the end of the article

\begin{abstract}
In this paper, we consider the following nonlinear boundary value problem: $\left(\varphi\left(u^{\prime}(t)\right)\right)^{\prime}+a(t) f(u(t))=0,0<t<1, u(0)=\sum_{i=1}^{m-2} \alpha_{i} u\left(\xi_{i}\right), u^{\prime}(1)=0$, where $\varphi: \mathbb{R} \rightarrow \mathbb{R}$ is an increasing homeomorphism and positive homomorphism with $\varphi(0)=0$. By using a fixed-point theorem on partially ordered sets, we obtain sufficient conditions for the existence and uniqueness of positive and nondecreasing solutions to the above boundary value problem.

MSC: 34B18; 34B27
\end{abstract}

Keywords: partially ordered sets; fixed-point theorem; positive solution

\section{Introduction}

In this paper, we consider the existence and uniqueness of a positive and nondecreasing solution to the following boundary value problem:

$$
\begin{aligned}
& \left(\varphi\left(u^{\prime}(t)\right)\right)^{\prime}+a(t) f(u(t))=0, \quad 0<t<1, \\
& u(0)=\sum_{i=1}^{m-2} \alpha_{i} u\left(\xi_{i}\right), \quad u^{\prime}(1)=0,
\end{aligned}
$$

where $\varphi: \mathbb{R} \rightarrow \mathbb{R}$ is an increasing homeomorphism and positive homomorphism with $\varphi(0)=0$. Here $\xi_{i} \in(0,1)$ with $0<\xi_{1}<\xi_{2}<\cdots<\xi_{m-2}<1$ and $\alpha_{i}$ satisfy $\alpha_{i} \in[0,+\infty), 0<$ $\sum_{i=1}^{m-2} \alpha_{i}<1$.

A projection $\varphi: \mathbb{R} \rightarrow \mathbb{R}$ is called an increasing homeomorphism and positive homomorphism, if the following conditions are satisfied:

(1) $\varphi(x) \leq \varphi(y)$, for all $x, y \in \mathbb{R}$ with $x \leq y$;

(2) $\varphi$ is a continuous bijection and its inverse mapping is also continuous;

(3) $\varphi(x y)=\varphi(x) \varphi(y)$, for all $x, y \in \mathbb{R}_{+}$.

In the above definition, we can replace the condition (3) by the following stronger condition:

(4) $\varphi(x y)=\varphi(x) \varphi(y)$, for all $x, y \in \mathbb{R}$, where $\mathbb{R}=(-\infty,+\infty)$.

2014 Miao et al.; licensee Springer. This is an Open Access article distributed under the terms of the Creative Commons Attribution License (http://creativecommons.org/licenses/by/2.0), which permits unrestricted use, distribution, and reproduction in any medium, provided the original work is properly cited. 
Remark 1.1 If conditions (1), (2), and (4) hold, then it implies that $\varphi$ is homogeneous generating a $p$-Laplace operator, i.e. $\varphi(x)=|x|^{p-2} x$, for some $p>1$.

Recently, the existence and multiplicity of positive solutions for the $p$-Laplacian operator, i.e., $\varphi(x)=|x|^{p-2} x$, for some $p>1$, have received wide attention, see [1-3] and references therein. We know that the oddness of a $p$-Laplacian operator is key to the proof. However, in this paper we define a new operator, which improves and generates a $p$ Laplacian operator for some $p>1$, and $\varphi$ is not necessarily odd. Moreover research of increasing homeomorphisms and positive homomorphism operators has proceeded very slowly, see $[4,5]$.

In [4], Liu and Zhang studied the existence of positive solutions of quasilinear differential equation

$$
\begin{aligned}
& \left(\varphi\left(x^{\prime}\right)\right)^{\prime}+a(t) f(x(t))=0, \quad 0<t<1, \\
& x(0)-\beta x^{\prime}(0)=0, \quad x(1)+\delta x^{\prime}(1)=0,
\end{aligned}
$$

where $\varphi: \mathbb{R} \rightarrow \mathbb{R}$ is an increasing homeomorphism and positive homomorphism and $\varphi(0)=0$. They obtain the existence of one or two positive solutions by using a fixed-point index theorem in cones. But the uniqueness of the solution is not treated.

In [5], the authors showed that there exist countably many positive solutions by using the fixed-point index theory and a new fixed-point theorem in cones. They also assumed that the operator $\varphi: \mathbb{R} \rightarrow \mathbb{R}$ is an increasing homeomorphism and a positive homomorphism, and $\varphi(0)=0$.

In [6], the authors established the existence and uniqueness of a positive and nondecreasing solution to a singular boundary value problem of a class of nonlinear fractional differential equation. Their analysis relies on a fixed-point theorem in partially ordered sets. The existence of a fixed point in partially ordered sets has been considered recently in $[6-10]$.

But whether or not we can obtain the existence and uniqueness of a positive and nondecreasing solution to the boundary value problem (1.1)-(1.2) still remains unknown. So, motivated by all the works above, we will prove the existence and uniqueness of a positive and nondecreasing solution for the boundary value problems (1.1)-(1.2) by using a fixed-point theorem on partially ordered sets.

\section{Some definitions and fixed-point theorems}

Definition 2.1 Let $(E,\|\cdot\|)$ be a real Banach space. A nonempty, closed, convex set $P \subset E$ is said to be a cone provided the following are satisfied:

(a) if $y \in P$ and $\lambda \geq 0$, then $\lambda y \in P$;

(b) if $y \in P$ and $-y \in P$, then $y=0$.

If $P \subset E$ is a cone, we denote the order induced by $P$ on $E$ by $\leq$, that is, $x \leq y$ if and only if $y-x \in P$.

The following fixed-point theorems in partially ordered sets are fundamental and important to the proofs of our main results.

Theorem 2.1 ([7]) Let $(E, \leq)$ be a partially ordered set and suppose that there exists a metric $d$ in $E$ such that $(E, d)$ is a complete metric space. Assume that E satisfies the following 
condition:

if $\left\{x_{n}\right\}$ is a nondecreasing sequence in $E$ such that $x_{n} \rightarrow x$, then $x_{n} \leq x, \forall n \in \mathbb{N}$.

Let $T: E \rightarrow E$ be a nondecreasing mapping such that

$$
d(T x, T y) \leq d(x, y)-\psi(d(x, y)), \quad \text { for } x \geq y,
$$

where $\psi:[0,+\infty) \rightarrow[0,+\infty)$ is a continuous and nondecreasing function such that $\psi$ is positive in $(0,+\infty), \psi(0)=0$ and $\lim _{t \rightarrow \infty} \psi(t)=\infty$. If there exists $x_{0} \in E$ with $x_{0} \leq T\left(x_{0}\right)$, then $T$ has a fixed point.

If we consider that $(E, \leq)$ satisfies the following condition:

$$
\text { for } x, y \in E \text { there exists } z \in E \text { which is comparable to } x \text { and } y \text {, }
$$

then we have the following result.

Theorem 2.2 ([8]) Adding condition (2.2) to the hypotheses of Theorem 2.1, we obtain uniqueness of the fixed point.

\section{Main results}

The basic space used in this paper is $E=C[0,1]$. Then $E$ is a real Banach space with the norm $\|u\|=\max _{0 \leq t \leq 1}|u(t)|$. Note that this space can be equipped with a partial order given by

$$
x, y \in C[0,1], \quad x \leq y \quad \Leftrightarrow \quad x(t) \leq y(t), \quad t \in[0,1] .
$$

In $[8]$ it is proved that $(C[0,1], \leq)$ with the classic metric given by

$$
d(x, y)=\sup _{0 \leq t \leq 1}\{|x(t)-y(t)|\}
$$

satisfies condition (2.1) of Theorem 2.1. Moreover, for $x, y \in C[0,1]$ as the function $\max \{x, y\} \in C[0,1],(C[0,1], \leq)$ satisfies condition (2.2).

The main result of this paper is the following.

Theorem 3.1 The boundary value problem (1.1)-(1.2) has a unique positive solution $u(t)$ which is strictly increasing if the following conditions are satisfied:

(A) $a(t)$ is a nonnegative measurable function defined in $[0,1]$ and $a(t)$ does not identically vanish on any subinterval of $[0,1]$ and

$$
0<\int_{0}^{1} a(t) d t<+\infty
$$

$\left(f_{1}\right) f:[0,+\infty) \rightarrow[0,+\infty)$ is continuous and nondecreasing respect to $u$ and $f(u(t)) \not \equiv 0$ for $t \in Z \subset[0,1]$ with $\mu(Z)>0$ ( $\mu$ denotes the Lebesgue measure); 
$\left(\mathrm{f}_{2}\right)$ there exists $1<\lambda+1<\frac{1-\sum_{i=1}^{m-2} \alpha_{i}}{\varphi^{-1}\left(\int_{0}^{1} a(\tau) d \tau\right)}$ such that for $u, v \in[0,+\infty)$ with $u \geq v$ and $t \in[0,1]$

$$
\varphi(\ln (v+2)) \leq f(v) \leq f(u) \leq \varphi\left(\ln (u+2)(u-v+1)^{\lambda}\right) .
$$

Proof Consider the cone

$$
K=\{u \in C[0,1]: u \geq 0\} .
$$

As $K$ is a closed set of $C[0,1], K$ is a complete metric space with the distance given by $d(u, v)=\sup _{t \in[0,1]}|u(t)-v(t)|$.

Now, we consider the operator $T$ defined by

$$
T u(t)=\int_{0}^{t} \varphi^{-1}\left(\int_{s}^{1} a(\tau) f(u(\tau)) d \tau\right) d s+\frac{\sum_{i=1}^{m-2} \alpha_{i} \int_{0}^{\xi_{i}} \varphi^{-1}\left(\int_{s}^{1} a(\tau) f(u(\tau)) d \tau\right) d s}{1-\sum_{i=1}^{m-2} \alpha_{i}} .
$$

By conditions $(\mathrm{A}),\left(\mathrm{f}_{1}\right)$, we have $T(K) \subset K$.

We now show that all the conditions of Theorem 2.1 and Theorem 2.2 are satisfied.

Firstly, by condition $\left(f_{1}\right)$, for $u, v \in K$ and $u \geq v$, we have

$$
\begin{aligned}
T u(t) & =\int_{0}^{t} \varphi^{-1}\left(\int_{s}^{1} a(\tau) f(u(\tau)) d \tau\right) d s+\frac{\sum_{i=1}^{m-2} \alpha_{i} \int_{0}^{\xi_{i}} \varphi^{-1}\left(\int_{s}^{1} a(\tau) f(u(\tau)) d \tau\right) d s}{1-\sum_{i=1}^{m-2} \alpha_{i}} \\
& \geq \int_{0}^{t} \varphi^{-1}\left(\int_{s}^{1} a(\tau) f(v(\tau)) d \tau\right) d s+\frac{\sum_{i=1}^{m-2} \alpha_{i} \int_{0}^{\xi_{i}} \varphi^{-1}\left(\int_{s}^{1} a(\tau) f(v(\tau)) d \tau\right) d s}{1-\sum_{i=1}^{m-2} \alpha_{i}} \\
& =T \nu(t) .
\end{aligned}
$$

This proves that $T$ is a nondecreasing operator. On the other hand, for $u \geq v$ and by $\left(\mathrm{f}_{2}\right)$ we have

$$
\begin{aligned}
d(T u, T v) & \\
= & \sup _{0 \leq t \leq 1}|(T u)(t)-(T v)(t)|=\sup _{0 \leq t \leq 1}((T u)(t)-(T v)(t)) \\
\leq & \sup _{0 \leq t \leq 1} \int_{0}^{t}\left[\varphi^{-1}\left(\int_{s}^{1} a(\tau) f(u(\tau)) d \tau\right)-\varphi^{-1}\left(\int_{s}^{1} a(\tau) f(v(\tau)) d \tau\right)\right] d s \\
& +\frac{\sum_{i=1}^{m-2} \alpha_{i} \int_{0}^{\xi_{i}}\left[\varphi^{-1}\left(\int_{s}^{1} a(\tau) f(u(\tau)) d \tau\right)-\varphi^{-1}\left(\int_{s}^{1} a(\tau) f(v(\tau)) d \tau\right)\right] d s}{1-\sum_{i=1}^{m-2} \alpha_{i}} \\
\leq & \varphi^{-1}\left(\int_{0}^{1} a(\tau) d \tau\right)\left(\ln (u+2)(u-v+1)^{\lambda}-\ln (v+2)\right) \\
& +\frac{\sum_{i=1}^{m-2} \alpha_{i} \xi_{i} \varphi^{-1}\left(\int_{0}^{1} a(\tau) d \tau\right)}{1-\sum_{i=1}^{m-2} \alpha_{i}}\left(\ln (u+2)(u-v+1)^{\lambda}-\ln (v+2)\right) \\
\leq & {\left[\varphi^{-1}\left(\int_{0}^{1} a(\tau) d \tau\right)+\frac{\sum_{i=1}^{m-2} \alpha_{i} \xi_{i} \varphi^{-1}\left(\int_{0}^{1} a(\tau) d \tau\right)}{1-\sum_{i=1}^{m-2} \alpha_{i}}\right]\left(\ln \frac{(u+2)(u-v+1)^{\lambda}}{v+2}\right) } \\
\leq & (\lambda+1) \ln (u-v+1) \frac{\varphi^{-1}\left(\int_{0}^{1} a(\tau) d \tau\right)}{1-\sum_{i=1}^{m-2} \alpha_{i}} .
\end{aligned}
$$


Since the function $h(x)=\ln (x+1)$ is nondecreasing, and condition $\left(\mathrm{f}_{2}\right)$, then we have

$$
\begin{aligned}
d(T u, T v) & \leq(\lambda+1) \ln (\|u-v\|+1) \frac{\varphi^{-1}\left(\int_{0}^{1} a(\tau) d \tau\right)}{1-\sum_{i=1}^{m-2} \alpha_{i}}<\ln (\|u-v\|+1) \\
& =\|u-v\|-(\|u-v\|-\ln (\|u-v\|+1)) .
\end{aligned}
$$

Let $\psi(x)=x-\ln (x+1)$. Obviously $\psi:[0,+\infty) \rightarrow[0,+\infty)$ is continuous, nondecreasing, positive in $(0,+\infty), \psi(0)=0$, and $\lim _{x \rightarrow+\infty} \psi(x)=+\infty$. Thus, for $u \geq v$, we have

$$
d(T u, T v) \leq d(u, v)-\psi(d(u, v))
$$

By conditions $(A)$ and $\left(f_{1}\right)$, we know that

$$
\begin{aligned}
(T 0)(t) & =\int_{0}^{t} \varphi^{-1}\left(\int_{s}^{1} a(\tau) f(0) d \tau\right) d s+\frac{\sum_{i=1}^{m-2} \alpha_{i} \int_{0}^{\xi_{i}} \varphi^{-1}\left(\int_{s}^{1} a(\tau) f(0) d \tau\right) d s}{1-\sum_{i=1}^{m-2} \alpha_{i}} \\
& \geq 0 .
\end{aligned}
$$

Therefore, by Theorem 2.1 we know that problem (1.1)-(1.2) has at least one nonnegative solution. As $(K, \leq)$ satisfies condition (2.2), thus, Theorem 2.2 implies the uniqueness of the solution. By definition of $T$ and conditions $(A),\left(f_{1}\right)$, it is easy to prove that this solution $u(t)$ is strictly increasing.

\section{Example}

Example 4.1 Consider the boundary value problem

$$
\begin{cases}\left(\varphi\left(u^{\prime}(t)\right)\right)^{\prime}+\frac{1}{5} t^{4} f(u(t))=0, & 0<t<1, \\ u(0)=\frac{1}{4} u\left(\frac{1}{4}\right)+\frac{1}{4} u\left(\frac{1}{2}\right), & u^{\prime}(1)=0,\end{cases}
$$

where

$$
\varphi(u)= \begin{cases}\frac{u^{3}}{1+u^{2}}, & u \leq 0 \\ u^{2}, & u>0\end{cases}
$$

$a(t)=\frac{1}{5} t^{4}$ and $f(x)=[\ln (x+2)]^{2}$ for $x \in[0,+\infty)$.

Proof Note that $f$ is a continuous function and $f(x)>0$. Moreover, $f$ is nondecreasing with respect to $x$ since $\frac{\partial f}{\partial x}=\frac{2}{x+2} \ln (x+2)>0$. On the other hand, for $u \geq v$, we have

$$
\begin{aligned}
\varphi(\ln (v+2)) & =[\ln (v+2)]^{2}=f(v) \leq f(u)=[\ln (u+2)]^{2} \\
& \leq(\ln (u+2)(u-v+1))^{2} \\
& =\varphi(\ln (u+2)(u-v+1)) .
\end{aligned}
$$

In this case, $\lambda=1$ because $1<\lambda+1<\frac{1-\sum_{i=1}^{m-2} \alpha_{i}}{\varphi^{-1}\left(\int_{0}^{1} a(\tau) d \tau\right)}=\frac{5}{2}$. Thus Theorem 3.1 implies that the boundary value problem (4.1) has a unique positive solution which is strictly increasing. 


\section{Competing interests}

The authors declare that they have no competing interests.

\section{Authors' contributions}

The authors contributed equally in this article. They read and approved the final manuscript.

\section{Author details}

${ }^{1}$ College of Mathematics, Changchun Normal University, Changchun, Jilin 130032, PR China. ${ }^{2}$ Scientific Research Department, Changchun Normal University, Changchun, Jilin 130032, PR China.

\section{Acknowledgements}

The authors would like to express sincere thanks to the anonymous referee for his/her carefully reading the manuscript and valuable comments and suggestions. The authors are supported by the National Natural Science Foundation of China (Grant No. 11301038), Research Foundation during the 12st Five-Year Plan Period of Department of Education of Jilin Province, China (Grant [2013] No. 252), Youth Foundation for Science and Technology Department of Jilin Province (20130522100JH).

\section{Received: 5 August 2013 Accepted: 17 December 2013 Published: 16 Jan 2014}

\section{References}

1. Bai, C, Fang, J: Existence of multiple positive solution for nonlinear $m$-point boundary value problems. Appl. Math. Comput. 140, 297-305 (2003)

2. Liu, B: Positive solutions three-points boundary value problems for one-dimensional $p$-Laplacian with infinitely many singularities. Appl. Math. Lett. 17, 655-661 (2004)

3. Wang, Y, Ge, W: Existence of multiple positive solutions for multi-point boundary value problems with a one-dimensional p-Laplacian. Nonlinear Anal. 67, 476-485 (2007)

4. Liu, BF, Zhang, JH: The existence of positive solutions for some nonlinear boundary value problems with linear mixed boundary conditions. J. Math. Anal. Appl. 309, 505-516 (2005)

5. Liang, $\mathrm{SH}$, Zhang, JH: The existence of countably many positive solutions for nonlinear singular $m$-point boundary value problems. J. Comput. Appl. Math. 214, 78-89 (2008)

6. Caballero Mena, J, Harjani, J, Sadarangani, K: Existence and uniqueness of positive and nondecreasing solutions for a class of singular fractional boundary value problems. Bound. Value Probl. 2009, Article ID 421310 (2009). doi:10.1155/2009/421310

7. Harjani, J, Sadarangani, K: Fixed point theorems for weakly contractive mappings in partially ordered sets. Nonlinear Anal. 71, 3403-3410 (2009)

8. Nieto, JJ, Rodríguez-López, R: Contractive mapping theorems in partially ordered sets and applications to ordinary differential equations. Order 22, 223-239 (2005)

9. Nieto, JJ, Rodríguez-López, R: Fixed point theorems in ordered abstract spaces. Proc. Am. Math. Soc. 135(8), 2505-2517 (2007)

10. O'Regan, D, Petrusel, A: Fixed point theorems for generalized contractions in ordered metric spaces. J. Math. Anal. Appl. 341, 1241-1252 (2008)

10.1186/1687-1847-2014-20

Cite this article as: Miao et al.: Existence and uniqueness of positive solutions to boundary value problem with increasing homeomorphism and positive homomorphism operator. Advances in Difference Equations 2014, 2014:20

\section{Submit your manuscript to a SpringerOpen ${ }^{0}$ journal and benefit from:}

- Convenient online submission

- Rigorous peer review

- Immediate publication on acceptance

- Open access: articles freely available online

- High visibility within the field

- Retaining the copyright to your article 